\title{
Star Formation at High Galactic Latitude: A Case for Extensions to the $\mathrm{H} \alpha$ Survey
}

\author{
Kristen A. Larson \\ Department of Physics, Applied Physics \& Astronomy, \\ Rensselaer Polytechnic Institute, Troy, NY 12180, USA \\ kal@charon.phys.rpi.edu
}

\begin{abstract}
The AAO/UKST H $\alpha$ Survey should be extended to high Galactic latitude $\left(|b|>25^{\circ}\right)$ to search for $\mathrm{T}$ Tauri stars. The $\mathrm{H} \alpha$ Survey can contribute to a complete inventory of young stellar objects in high-latitude clouds, which will help define the limits of conditions and processes that lead to star formation.
\end{abstract}

Keywords: ISM: clouds — stars: emission-line — stars: pre-main sequence — surveys

\section{High-latitude Clouds}

Until recently, most studies of star formation have been confined to large molecular clouds near the Galactic plane, such as Taurus and Orion. However, both greater sensitivity of the receivers detecting radio emission lines as well as the all-sky surveys of IRAS and later satellites have made possible the discovery and study of more tenuous molecular material. Although these clouds are thought to exist throughout the Galaxy, they are most easily observed at high latitude above the molecular scale height where sightlines are particularly simple. Over a hundred high-latitude molecular clouds (HLCs) have been found in regions obscured on photographic plates and in regions of $100 \mu \mathrm{m}$ excess emission due to dust associated with molecular gas. HLCs exhibit a wide range of morphologies and sizes, from small dense clumps to filaments several degrees long, and spatial structure down to the smallest observable scales. In general, HLCs are translucent ( $\left.A_{\mathrm{V}} \sim 1 \mathrm{mag}\right)$, located within a few hundred parsecs of the Sun, quiescent and not gravitationally bound (see the review in Magnani et al. 1996).

In an effort to constrain models of the evolution of dust in the interstellar medium, we are currently observing interstellar extinction and polarisation curves toward HLCs. Preliminary results suggest that, in general, the dust in HLCs has optical properties not unlike dust observed in the Galactic plane. However, we have found a large variation in the environmentally-sensitive relationship between polarisation and extinction within and among HLCs. We are currently investigating how the polarisation efficiency $(p / A)$ and the ratio of total-to-selective extinction $\left(R_{\mathrm{V}}\right)$ in HLCs is correlated with the presence of $\mathrm{T}$ Tauri stars at high latitude.

\section{Star Formation in HLCs}

Given that most high-latitude clouds are translucent and quiescent, it is somewhat surprising that several have been found to contain young stellar objects. Furthermore, several high-latitude young stellar objects have been found serendipitously, apparently isolated from any molecular material (Feigelson 1996). Since these discoveries, all-sky databases from missions such as IRAS and Einstein, have been searched for point sources having the infrared and $\mathrm{X}$-ray spectral signatures of T Tauri stars, yielding hundred of candidates (e.g. Magnani et al. 1995; Caillault, Magnani \& Fryer 1995). However, the most convincing signature of stellar youth is $\mathrm{H} \alpha$ emission from the interface of the stellar photosphere and circumstellar material. Most high-latitude $\mathrm{H} \alpha$ surveys to date have been objective prism images of well-known CO clouds (e.g. Kun 1992). When digitised and paired with continuum images, the new AAO/UKST H $\alpha$ Survey will have the advantages of increased area, resolution, and sensitivity.

Gravitational collapse of cores in dense clouds is known to form stars. By what mechanism could T Tauri stars form in translucent HLCs? Possibilities include shock-induced formation, dispersal/ejection from other regions, and formation in situ followed by evaporation of the surrounding cloud. When compared with multi-wavelength measurements of cloud environment, the detection of T Tauri stars at high-latitude will have important implications for our understanding of the limits of conditions and processes that lead to star formation. To date, none of the T Tauri stars found at high latitude are in a translucent cloud environment, but a more systematic search is needed. 


\section{Extending the AAO/UKST H $\alpha$ Survey}

Given both the incomplete nature of surveys for HLCs as well as the existence of completely isolated T Tauri stars, the search for point-source emissionline objects at high latitude requires an unbiased survey. I propose that the AAO/UKST $\mathrm{H} \alpha$ survey be extended out from the Galactic plane to include fields of known HLCs as well as fields with no previous $\mathrm{CO}$ observations. $\mathrm{H} \alpha$ emission is one of the most proven and reliable spectral signatures of T Tauri stars, making the AAO/UKST H $\alpha$ Survey well poised to contribute to our understanding of star formation in the high-latitude environment.

\section{Acknowledgments}

K.A.L. thanks the Astronomer-in-Charge and staff of the AAO Schmidt Telescope for generous hospitality and support during this research project and in preparation of this manuscript.

Caillault, J.-P., Magnani, L., \& Fryer, C. 1995, ApJ, 441, 261

Feigelson, E. D. 1996, ApJ, 468, 306

Kun, M. 1992, A\&AS, 92, 875

Magnani, L., Caillault, J.-P., Buchalter, A., \& Beichman, C. A. 1995, ApJS, 96, 159

Magnani, L., Hartmann, D., \& Speck, B. G. 1996, ApJS, 106,447 